\title{
Westward drift in secular variation of the main geomagnetic field inferred from IGRF
}

\author{
Zi-Gang Wei and Wen-Yao Xu \\ Institute of Geology and Geophysics, Chinese Academy of Sciences, Beijing, 100029, China
}

(Received September, 17, 2002; Revised February 24, 2003; Accepted March 4, 2003)

\begin{abstract}
Westward drift is apparent not only in the Main Geomagnetic field (MG-field) but also in its Secular Variation (SV-field). The eighth generation of International Geomagnetic Reference Field (IGRF) is used in this paper to study westward drift in the SV-field. The magnetic potential of the SV-field shows a simple spatial distribution and steady variation tendency. An average westward drift rate ( 0.43 degree/year) is obtained from the SV potential for 1900-2005, which is much greater than the slow westward drift of the MG-field itself (about 0.15 degree/year) for the same period. Magnetic components $Y$ and $Z$ of the SV-field show complicated patterns, from which the average rates of westward drift are roughly estimated as 0.39 degree/year and 0.43 degree/year, respectively. The spatial distribution of component $X$ shows much more complicated pattern with many small-scale vertices, especially for the period 1940-1960, giving a larger drift rate (0.51 degree/year). The unusual behaviors of the high-degree Gauss coefficients in IGRF 1945, 1950 and 1955 slightly affect the spatial pattern of the potential, although they greatly distort the distribution of components $X, Y$ and $Z$.
\end{abstract}

\section{Introduction}

The westward drift is one of the most well known features in the Main Geomagnetic field (MG-field for short). Since Halley (1692) discovered this phenomenon and proposed the model of relative rotation between two spheres to interpret the westward drift, extensive analyses have been made on westward drift using various data and methods, and the Halley's model has undergone many refinements. Different results have been obtained for different epochs and different components of the field by different researchers, although an average westward drift velocity 0.2 degree/year is widely accepted (Bullard et al., 1950; Yukutake, 1962, 1981; Malin, 1969; Malin and Saunder, 1973; Jault et al., 1988; Tank, 2000). Using the eighth generation of IGRF and Briggs method (Briggs, 1968a, b), Wei and Xu (2000, 2001, 2002) obtained the average drift rate of 0.15 degree/year in the MG-field for last century.

Westward drift has been detected not only in the MGfield itself, but also in its first time derivative Secular Variation field (SV-field) (Bullard et al., 1950; Yukutake, 1968; Yukutake and Tachinaka, 1968; Langel, 1987). The drifts of the SV-field have been also extensively studied. The results show that the SV-field drifts westward at a much faster rate than the MG-field itself. For example, Bullard et al. (1950) compared the charts at various years, and estimated the westward drift rate of the SV-field as 0.32 degree/year. Yukutake (1968) obtained a similar rate 0.32 degree/year, while Yukutake and Tachinaka (1968) got a smaller rate $0.22-0.32$ degree/year.

The difference between the drift rates of the MG-field

Copy right(c) The Society of Geomagnetism and Earth, Planetary and Space Sciences (SGEPSS); The Seismological Society of Japan; The Volcanological Society of Japan; The Geodetic Society of Japan; The Japanese Society for Planetary Sciences. and SV-field is expected, since the drift of the MG-field is for explaining secular variation of the main field, or the first time derivative of the main field, as Malin and Saunder (1973) and Tank (2000) did; while the drift of the SV-field is for explaining variation of the SV-field, or the second time derivative of the main field, as Yukutake (1968) did. In the present work, we intend to study the global SV-field drift by using the eighth generation of IGRF models.

Different authors have their own work basis. Bullard et al. (1950) separated the drift rates of the dipole and non-dipole parts of the geomagnetic field, Malin (1969) determined the drift rate separately for each order of the spherical harmonic coefficients, Yukutake (1962) and Malin (1969) allowed the drift rate to vary with latitude, Yukutake (1962) and Matsushima and Honkura (1988) separated the secular variation into drifting and standing parts. Our idea in this paper is to consider the SV-field as a "moving random pattern", which is assumed to have a certain mean velocity and also to change randomly in form as it moves. It is different with the separation of moving and standing parts, as Yukutake (1962) did, and also different with pure rotation about an optimum pole (or, equivalently, an optimum axis), as Malin and Saunder (1973) and Tank (2000) did. These differences will lead to different physical explanations.

The drift velocity of a "moving random pattern" can be estimated by using Briggs' method (Briggs, 1968a, b). Although the Briggs' method is based on the identical principle, least squares, as other optimization methods did, it has some advantages on the stability and robustness of the computation.

In Section 2 of this paper, the general characteristics of the SV-field are studied by using the eighth generation of IGRF, the westward drift velocity of SV-field are calculated on the basis of magnetic potential in Section 3, the effects of the 

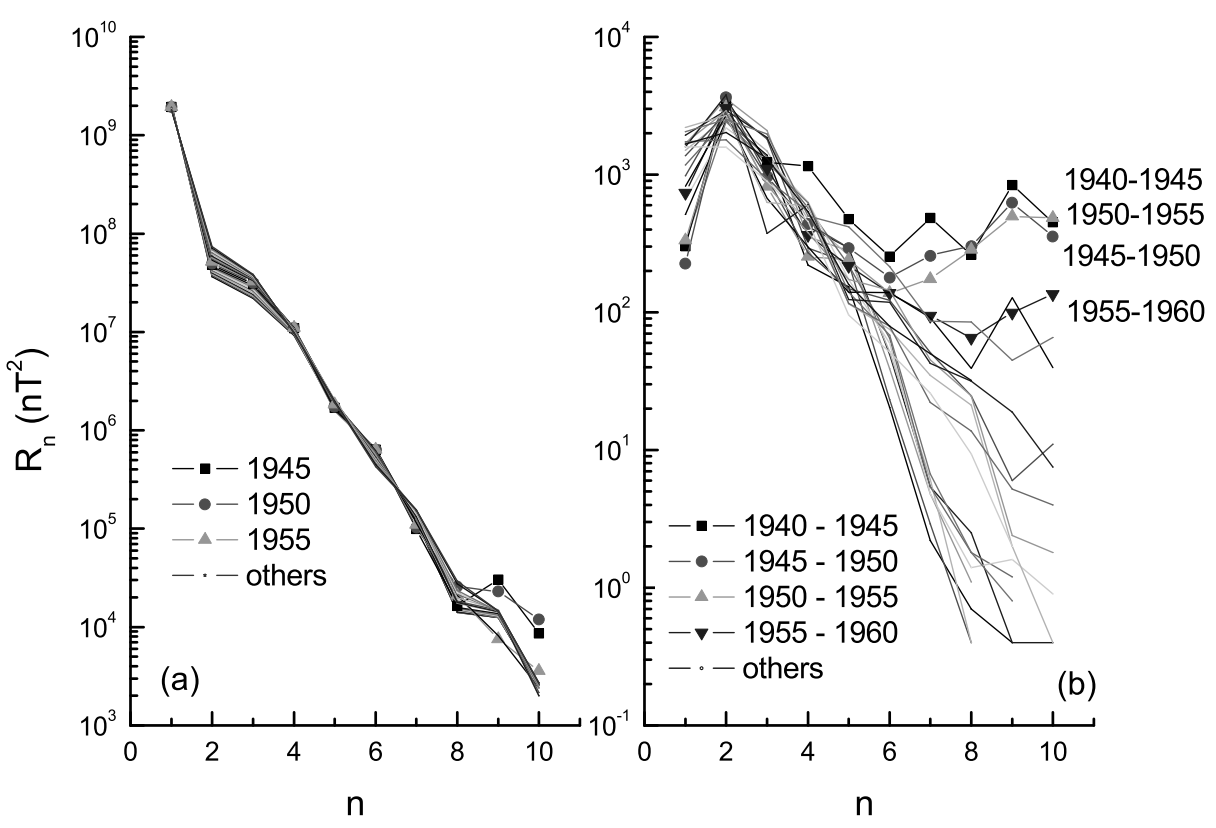

Fig. 1. Spectra of the MG-field (a) and SV-field (b).
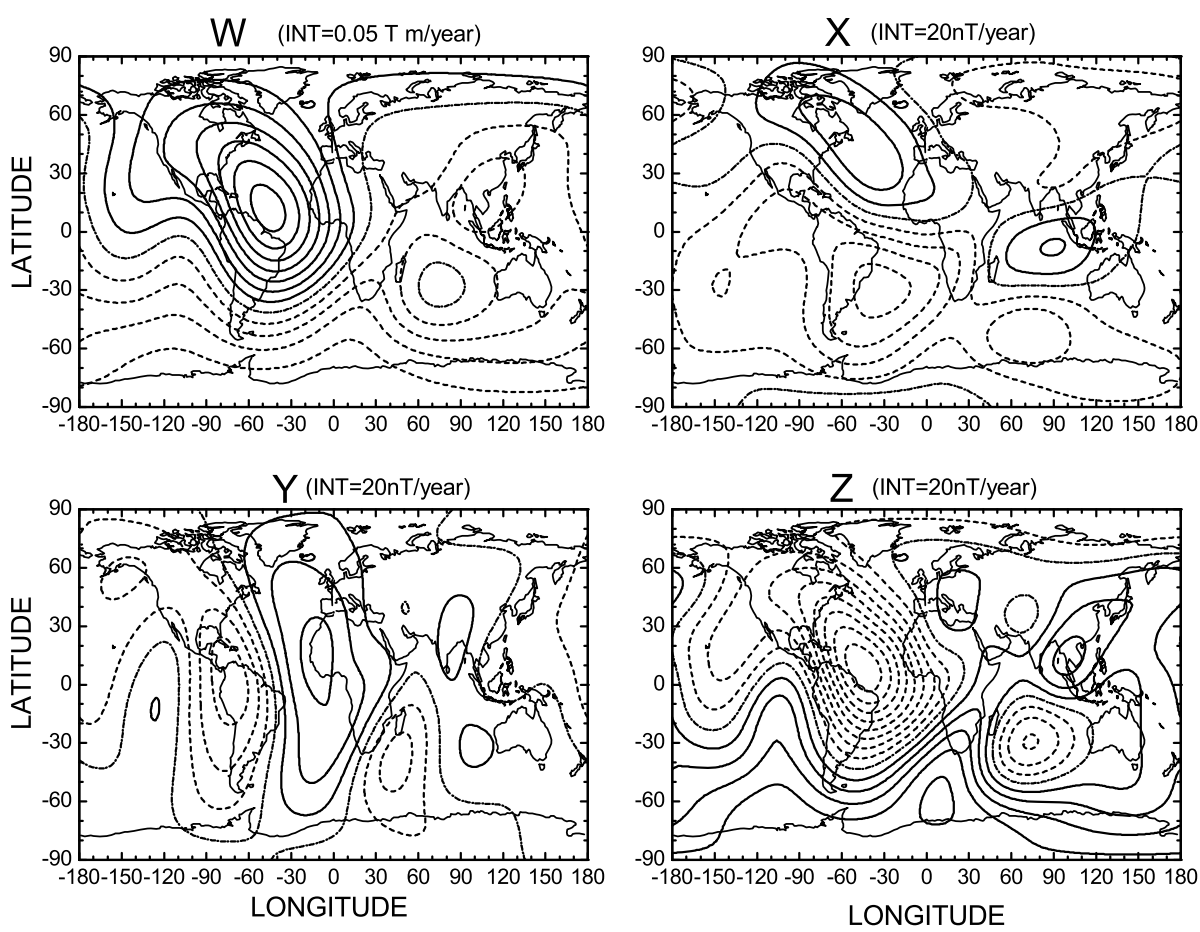

Fig. 2. Secular variations of the magnetic potential $W$ and the magnetic components $X, Y$ and $Z$, for 1990-1995. The solid (or dashed) lines represent increase (or decrease) of the field, the dot-dash lines represent zero variations of the field.

unusual features in IGRF1945-1955 on SV-field and its drift estimation are tested in Section 4. The differences among the results obtained from magnetic potential and magnetic components are also discussed.

\section{General Characteristics of the SV-Field}

The eighth generation of IGRF comprises 21 main geomagnetic field models at epochs 5 years apart from 1900 to 2000; and a SV model for 2000-2005 (IAGA Division 5, Working Group 8, 2000; Mandea and Macmillan, 2000). Each main-field model has 120 spherical harmonic (or Gauss) coefficients, $g_{n}^{m}$ and $h_{n}^{m}$, and extends to degree and order 10. The secular-variation model has 80 coefficients and extends to degree and order 8 . The geomagnetic potential in the IGRF models is represented in a series expansion (Langel, 1987)

$$
W=a \sum_{n=1}^{N} \sum_{m=0}^{n}\left(\frac{a}{r}\right)^{n+1}\left(g_{n}^{m} \cos m \lambda+h_{n}^{m} \sin m \lambda\right) P_{n}^{m}(\cos \theta)
$$




\section{SECULAR VARIATION OF THE SV-POTENTIAL} (INT: $0.05 \mathrm{~T} \mathrm{m/a)}$
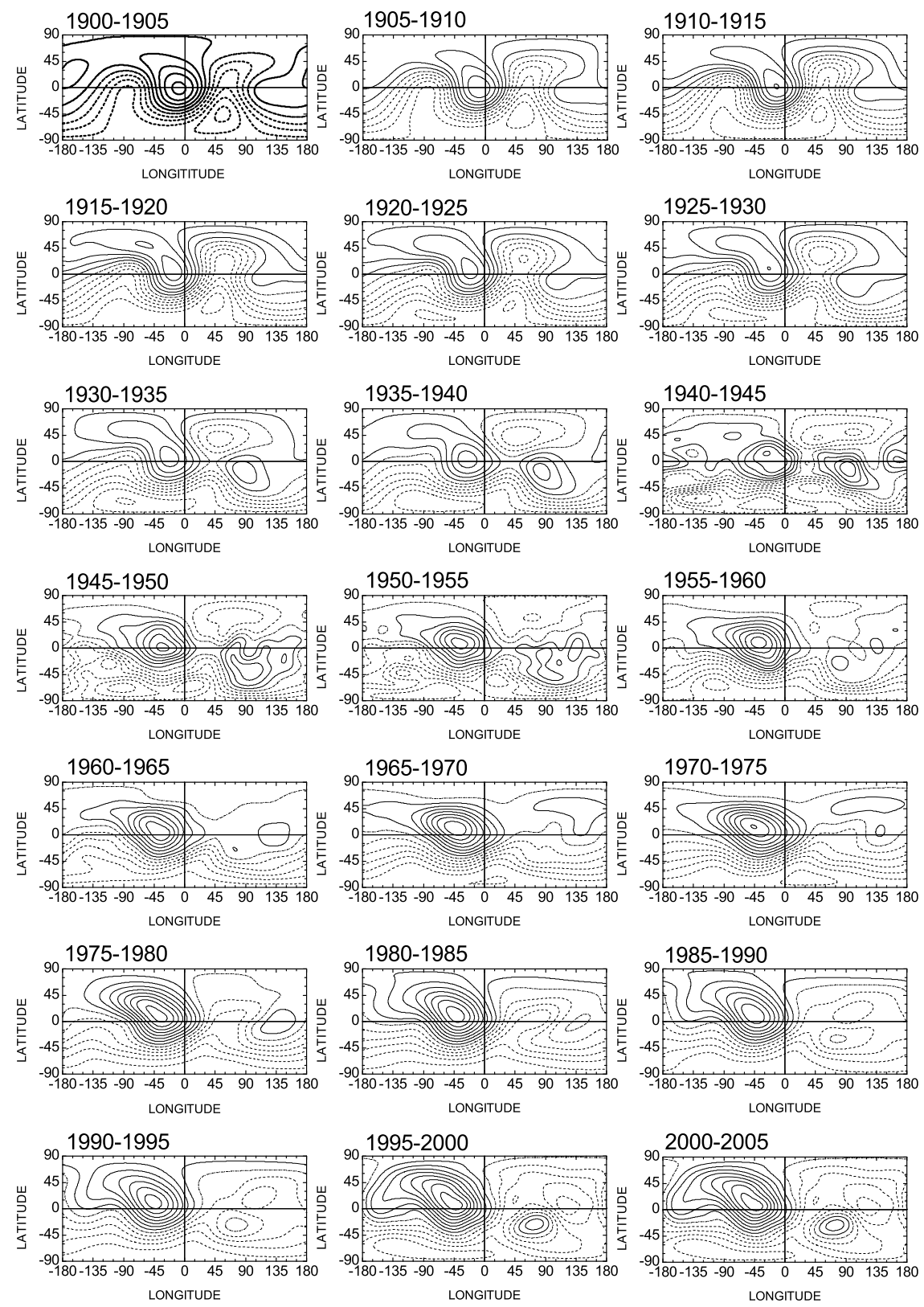

Fig. 3. Variations of the SV-potential during 1900-2005.

where $a$ is the mean radius of the Earth, $r$ is radial distance from the Earth's center, $\lambda$ is longitude eastward from Greenwich, $\theta$ is colatitude $\left(\theta=90^{\circ}-\phi, \phi\right.$ is latitude), and $P_{n}^{m}(\cos \theta)$ is the associated Legendre function of degree $n$ and order $m$, normalized according to the convention of Schmidt. $N$ is the maximum spherical harmonic degree of the expansion, or truncation level.

The IGRF models are used in this paper to calculate secular variations of the main field for each 5 -year interval by $\dot{g}_{n}^{m}(t)=\left(g_{n}^{m}(t+5)-g_{n}^{m}(t)\right) / 5$ and $\dot{h}_{n}^{m}(t)=$ $\left(h_{n}^{m}(t+5)-h_{n}^{m}(t)\right) / 5$. The SV-field shows different characteristics in comparison with the MG-field, implying that the secular variation of the main field is not simply decrease or increase in its intensity. A major difference between the SV-field and the MG-field is in their spectra $R_{n}=$ $(n+1) \sum_{m=0}^{n}\left[\left(g_{n}^{m}\right)^{2}+\left(h_{n}^{m}\right)^{2}\right]$ (Langel, 1987), as shown in

Fig. 1. In the MG-field spectrum (Fig. 1(a)) the magnitudes of high-degree harmonics are less than the dominant dipole by some 5 orders of magnitude, having unimportant contribution to whole field in the Earth's surface, but they are very important for understanding processes in the core, and to some extent for processes in the crust. In the spectrum of the SV-field (Fig. 1(b)), however, the quadrupole $(n=2)$ is dominant, while high-degree harmonics have magnitudes similar to the dipole terms, and are less than the dominant quadrupole by only order 2 of magnitude.

The differences between the SV-field and MG-field also exhibit in their spatial distribution. Figure 2 shows a typical example of the SV-field for 1990-1995, and the SV-fields for 


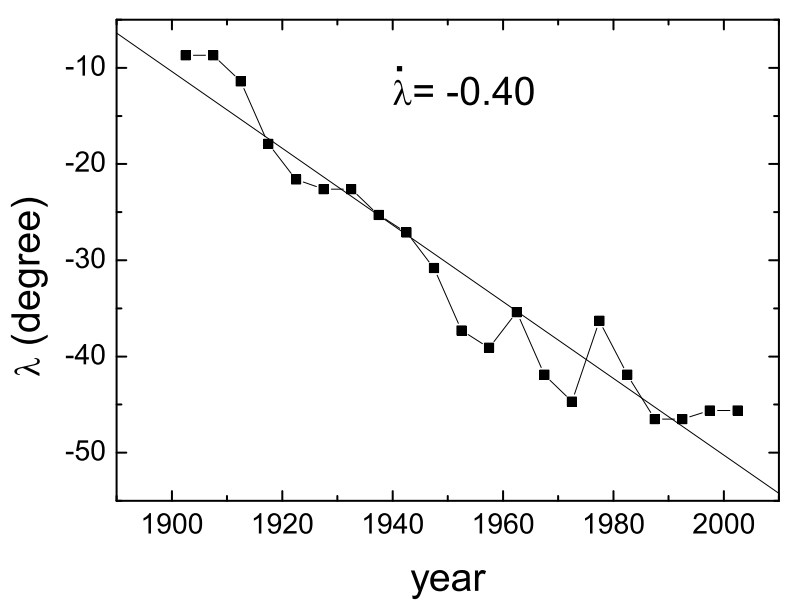

Fig. 4. Westward drift of the SV-potential estimated from Atlantic SV focus.

other 5-year intervals have similar characteristics. It is noted in the figure that the magnetic potential $W$ shows relatively simple spatial distribution with a dominant positive SV center in the central Atlantic and a minor negative SV center in Indian Ocean, implying dramatic SV in the western hemisphere and insignificant change in the eastern hemisphere. This contrast of E- and W-hemispheres is one of the principal features in the SV of the main field.

The contour maps of components $X, Y$ and $Z$ show more complicated patterns with several small-scale vertices, since the magnetic components are gradient of the magnetic potential, high-degree harmonics have more contribution than in the potential. Nevertheless, the correspondences of the components with the potential are obvious: $X$ contour map shows a positive center in northern Atlantic and a negative center in southern Atlantic, $Y$ contour has a positive center in eastern Atlantic and a negative center in western Atlantic, while $Z$ contour has a major negative center in Atlantic. The $\mathrm{SV}$ contour maps for the three components also show the dramatic variations in the western hemisphere and smaller changes in eastern hemisphere.

\section{Westward Drift of the SV-Field}

Westward drift rates of the magnetic field deduced from the magnetic components $X, Y$ and $Z$ are usually different (Bullard et al., 1950). Langel (1987) and Jault et al. (1988) analyzed the reasons of the differences. As Langel (1987) argued, the drift is much more apparent in the $Y$ component than in the $X$ or $Z$ components, partly because contours of equal $Y$ and $\dot{Y}$ tend to lie in the meridian plane. Another reason is that the symmetric portion of the field, described by the $g_{n}^{0}$ and $\dot{g}_{n}^{0}$ coefficients, cannot take part in a simple westward drift. This symmetric portion of the field is absent in the $Y$ component but is strong in the $X$ or $Z$ components and tends to mask any westward drift, which is present.

In this paper we use magnetic potential $W$ of the SV-field to calculate its westward drift rate, since the SV-potential has simple and stable structure in comparison with magnetic components, as mentioned above. Besides, it is expected that using the magnetic potential to calculate drift rate may avoid possible difficulties and confusion caused by the complicated pattern of the field components and different results from different components.

As mentioned above, the large SV in the western hemisphere and small SV in the eastern hemisphere implies that the westward drift of SV-field is largely dependent on the drift of the Atlantic region. In the following, we will examine the drift of the Atlantic SV center.

Figure 3 illustrates SV-fields for each 5-year interval during 1900-2005. It is noted that the positive Atlantic SV center is dominant in SV-field potential, and steadily drifts westward in the whole century.

In order to estimate the drift rate of the field, the variation of the longitude of the Atlantic SV center is plotted against time, as shown in Fig. 4. In the figure the time variation of the SV center is fitted by a straight line, the slop of which represents the average drift rate with negative (or positive) value for westward (or eastward) drift. From these data an average westward drift rate are estimated as 0.4 degree/year.

It should be pointed out that the unusual jumps in highdegree Gauss coefficient of IGRF 1945-1955 (Barton, 1997; $\mathrm{Xu}, 2000,2002)$ seriously distort the contour maps of $X, Y$ and $Z$ components, and make it difficult to accurately estimate drift rate (see Fig. 7 in the next section). However, these jumps have less significant effects on the contour structures of the potential $W$, accordingly, they have no essential effects on the estimation of westward drift.

Briggs $(1968 \mathrm{a}, \mathrm{b})$ proposed a method for estimating the drift of a moving random pattern, which moves with an average velocity and changes randomly in form as it moves. Assuming $f(t, \boldsymbol{r})$ and $f(t+\tau, \boldsymbol{r})$ are two frames of the pattern at instants $t$ and $t+\tau$, one can determine the displacement (or drift) of the pattern during this time span when the following correlation coefficient reaches its maximum value $\rho_{\max }$,

$$
\rho(t, \tau, \Delta \boldsymbol{r})=\frac{<\Delta f_{1} \Delta f_{2}>}{\sqrt{<\Delta f_{1}^{2}>} \sqrt{<\Delta f_{2}^{2}>}}
$$

where $\Delta f_{1}=f(t, \boldsymbol{r})-<f(t, \boldsymbol{r})>, \Delta f_{2}=$ $f(t+\tau, \boldsymbol{r}+\boldsymbol{\Delta} \boldsymbol{r})-<f(t+\tau, \boldsymbol{r}+\boldsymbol{\Delta} \boldsymbol{r})>$. $\boldsymbol{r}$ is location vector, the function with ' $<>$ ' means the spatial mean value on the earth surface. With a certain $\tau$, the correlation coefficient $\rho$ is calculated for various $\Delta \boldsymbol{r}$. The $\boldsymbol{\Delta} \boldsymbol{r}$ for the maximum $\rho$ (record it as $\rho_{\max }$ ) is considered as the displacement of the pattern during the time interval $\tau$, and the average drift velocity is $\Delta \boldsymbol{r} / \tau$. It is obvious that large $\rho_{\max }$ means insignificant random change of the pattern, while small $\rho_{\max }$ will be obtained for complicated and remarkable changes in the pattern. This will be discussed in Section 4 (see Fig. 8).

The geomagnetic isoline charts for two epochs $t$ and $t+\tau$ are regarded as two frames $f(t, r)$ and $f(t+\tau, \boldsymbol{r})$ of a moving random pattern. The latter frame $f(t+\tau, \boldsymbol{r})$ may be obtained from the early frame $f(t, r)$ by a displacement $\Delta \boldsymbol{r}$, which has a latitudinal component $\Delta \phi$ and a longitudinal component $\Delta \lambda$. In this way the global average drift velocity $\Delta r / \tau$ with a northward component $\Delta \phi / \tau$ and eastward component $\Delta \lambda / \tau$ can be obtained.

Considering the possible north-southward drift, we calculate the correlation coefficient $\rho$ of two frames in a latitude range of $\phi=-\left(90^{\circ}-\phi^{\prime}\right) \sim\left(90^{\circ}-\phi^{\prime}\right)$, instead of $\phi=-90^{\circ} \sim 90^{\circ}$. In our calculation the time span be- 

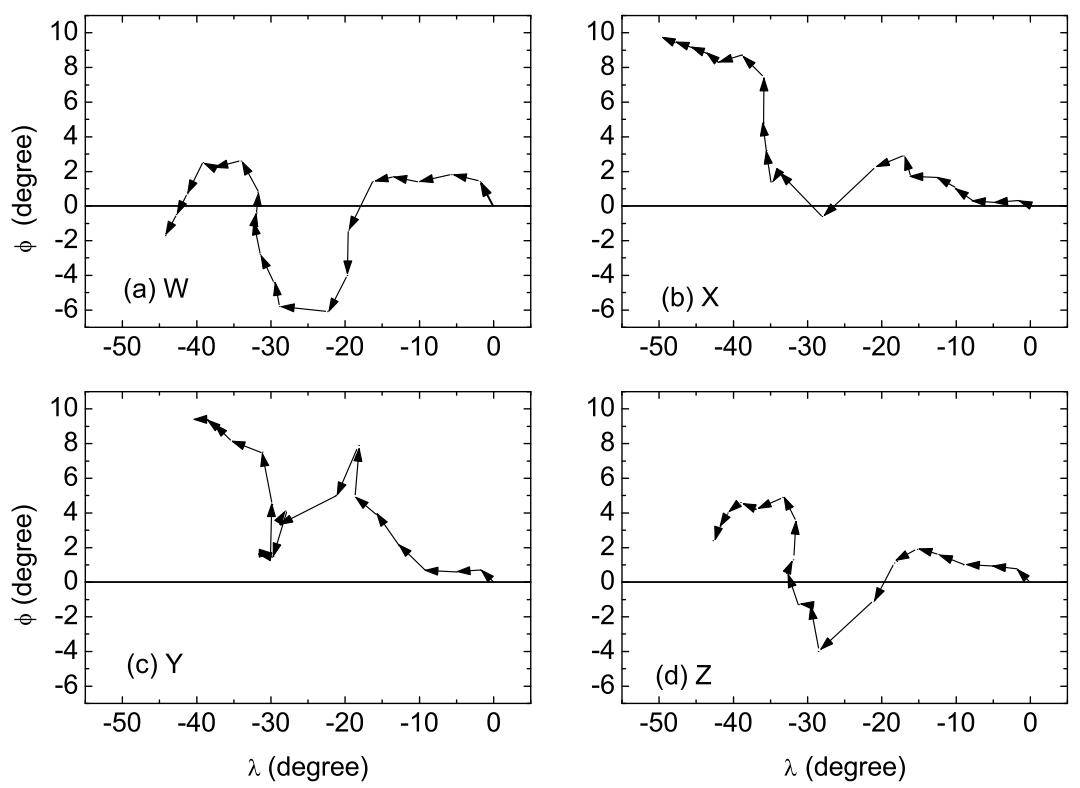

Fig. 5. Drift loci obtained from the SV-potential $W$ and the components $X, Y$ and $Z$ for 1900-2000.
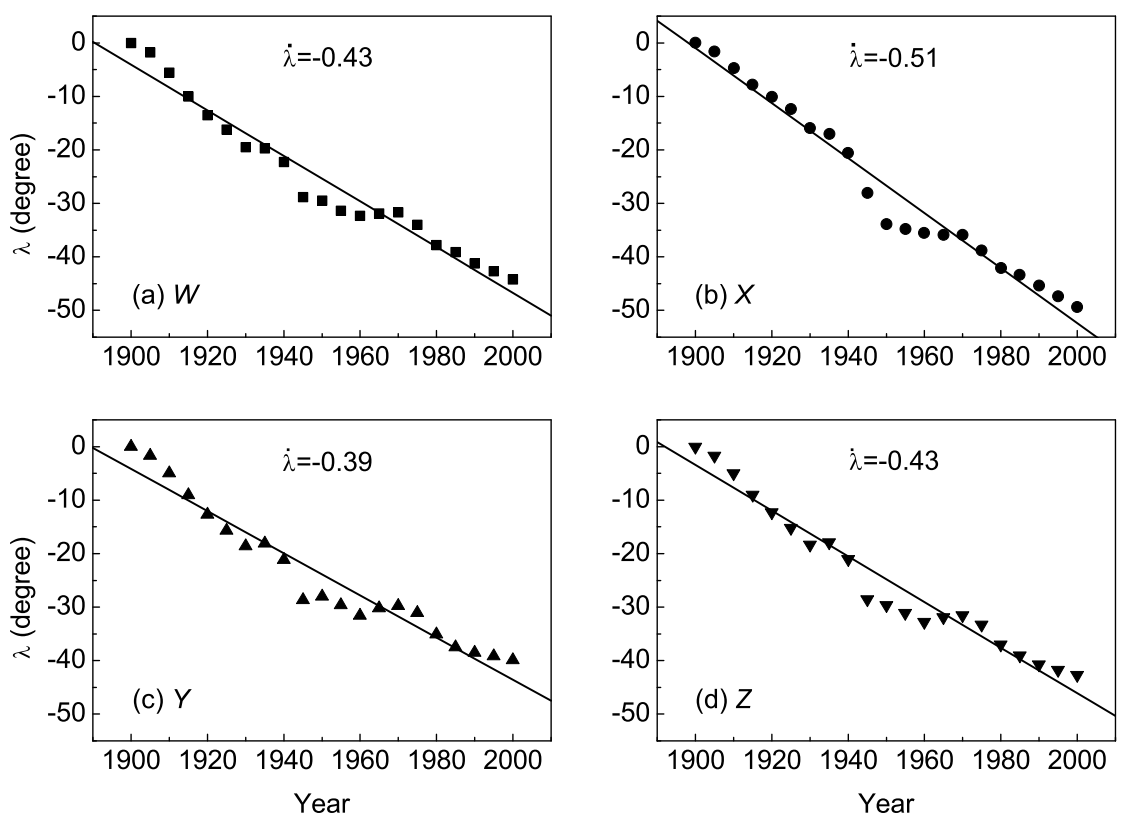

Fig. 6. Successive westward drifts against time for the SV-potential $W$ and the field components $X, Y$ and $Z$.

tween two frames is 10 years, the obtained maximum northsouthward drift is less than $4^{\circ}$, therefore, it should be reasonable to choose $\phi^{\prime}=5^{\circ} \sim 10^{\circ}$. In this paper $\phi^{\prime}=10^{\circ}$ is used. The error caused by ignoring the contribution from the polar cap fields is not essential, because the area of the polar caps is very small in comparison with the whole globe.

From the obtained drift velocities, the drift loci from 1900 to 2000 can be drawn, as depicted in Fig. 5, where the location for 1900 is taken as the start point.

It is noted in Fig. 5(a) that the SV-potential $W$ steadily drifts westward and oscillates north-southward. Figures 5(b), (c) and (d) for the magnetic components $X, Y$ and $Z$ show similar westward drift tendency, although the northsouthward drift is more or less different.

In Fig. 6 the westward drifts are plotted against time. The potential $W$ and the component $Z$ give similar estimations of the westward drift rate ( 0.43 degree/year). A little smaller rate, 0.39 degree/year, is obtained from the component $Y$, while a larger rate, 0.51 degree/year is obtained from the component $X$.

\section{Discussion and Conclusion}

Westward drifts of the MG-field and its SV-field are usually deduced from the magnetic components $X, Y$ and $Z$, or declination $D$. It can also be deduced from the magnetic potential $W$ (Langel, 1987). In the ideal case, they should give the same results. However, different components and potential show different drift (Langel, 1987; Jault et al., 1988). The essential point is because each of the four quantities gives different weight to the different harmonic coefficients 

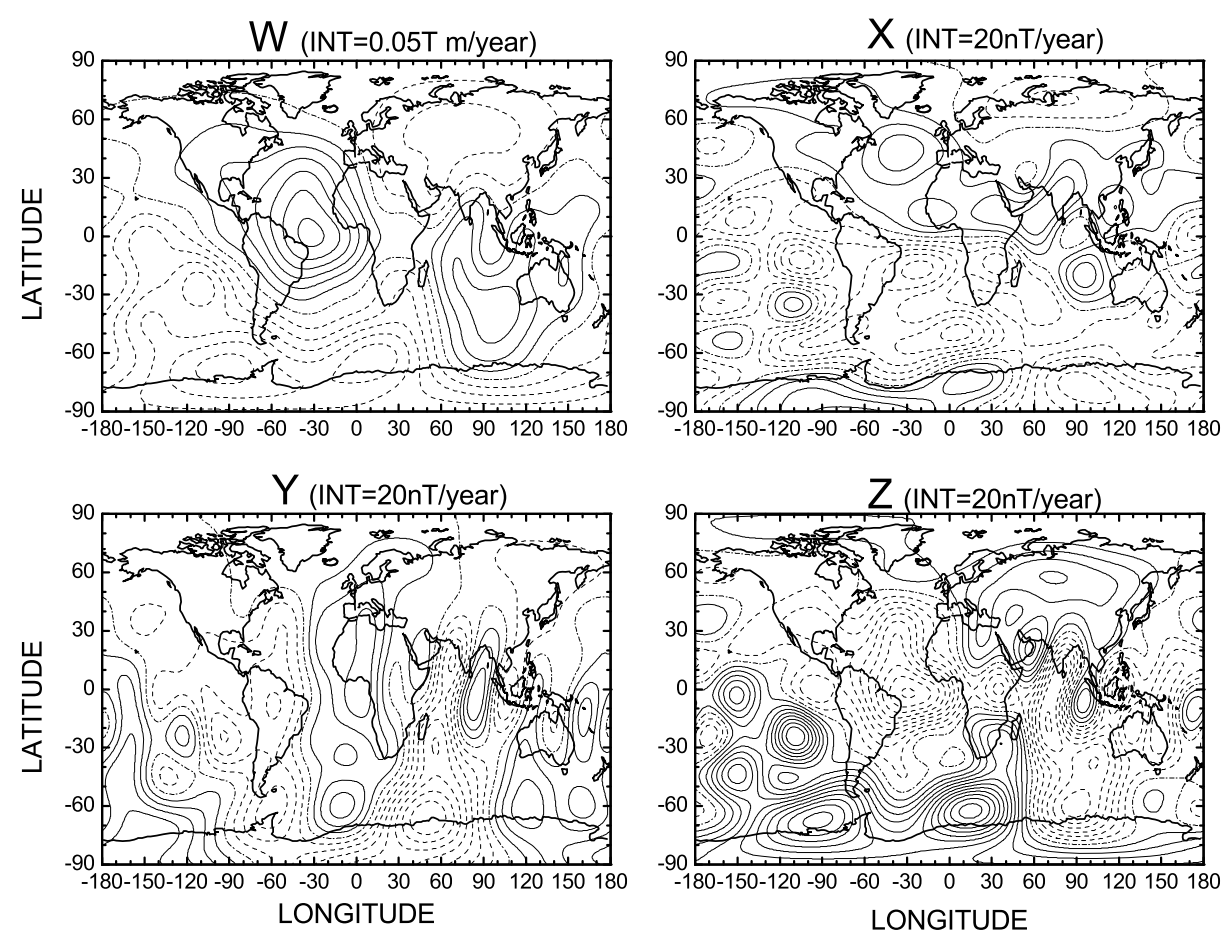

Fig. 7. Secular variations of the SV-potential $W$ and components $X, Y$ and $Z$ for 1945-1950

(see Jault et al., 1988). Using field components gives more weight to higher-degree coefficients than does using the potential. Using Gauss coefficients to calculate westward drift is, in fact, equivalent to using magnetic potential at the surface grids.

The magnetic potential has certain merits in comparison with the magnetic components in estimating westward drift rate: (1) The different drift rates obtained from different magnetic components raises some difficulties in uniquely determine the westward drift rate. It is not straightforward to give proper weights to each component. The same weights to each component were used in some cases, but it seems to be less logical (Yukutake, 1981). However, there is unique potential for MG-field or SV-field, and therefore, the drift rate of the field can be uniquely determined. (2) The magnetic potential $W$ shows relatively simple and stable spatial structure. However, magnetic components usually have more complicated contour maps, since magnetic components are the gradient of the potential. Unusual behaviors in IGRF models (for instance, IGRF1945-1955, (Xu, 2000, 2002)) will seriously distort the distribution of the component fields, but they have less significant effects on the magnetic potential. Figures 7(a), (b), (c) and (d) show respectively the SV of the potential $W$ and the components $X, Y$ and $Z$ for 1945 1950. It is noted in the figure that the magnetic potential $W$ maintains a spatial distribution very similar to that in Fig. 1 for normal cases. However, the patterns of three components show much more complicated structure than those in Fig. 1, which make it difficult to trace westward drift either of individual SV-field center or the whole pattern.

In order to show the above-mentioned difference between the components and potential, Fig. 8 shows $\rho_{\max }$ obtained from the SV-potential $W$ and the components $X, Y$ and $Z$, which are used to estimate the drift rate in Briggs method. It

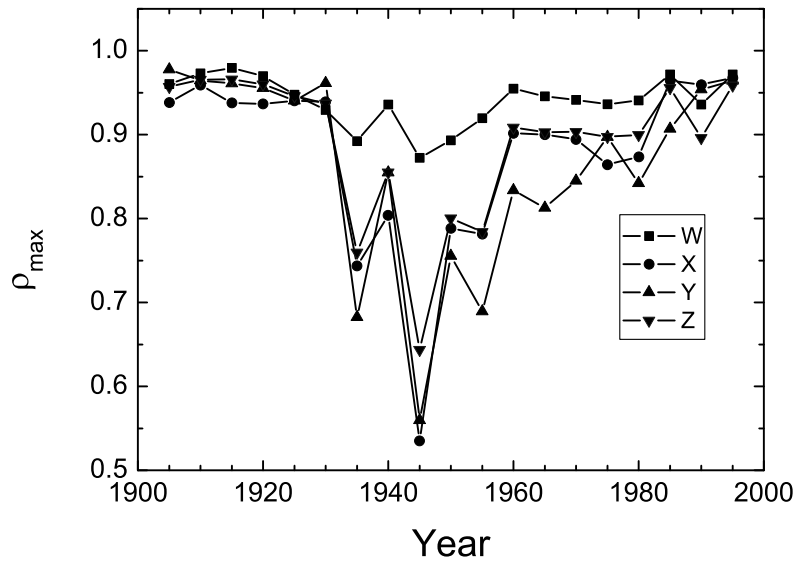

Fig. 8. $\rho_{\max }$ for the SV-potential $W$ and the components $X, Y$ and $Z$.

is clearly seen that the magnitudes of $\rho_{\max }$ obtained from the components $X, Y$ and $Z$ are much less than those from the potential $W$ for most epochs, especially for 1935-1960. In the absence of a satisfactory method for estimating the standard errors of drift, we can use $\rho_{\max }$ to give some qualitative statement. Large $\rho_{\max }$ implies less random deformation in the patterns and well-determined drift rate.

The main results in this paper are summarized as following:

(1) The SV-potential shows a stable spatial structure and a continuous variation with a steady westward drift at an average rate 0.43 degree/year during the period 1900-2005. The SV-field drifts much faster than the MG-field itself (about 0.15 degree/year) for the same period.

(2) The magnetic components $X, Y$ and $Z$ give diverging drift rates $0.51,0.39$ and 0.43 degree/year, respectively. 
(3) Since the SV-potential shows more steady and simple spatial distribution than the components, the estimate of drift by using the magnetic potential has certain merits over the components, especially, for those periods, when unusual behaviors in IGRF models heavily distort the component patterns.

Acknowledgments. The authors gratefully acknowledge the valuable remarks of the referees, Ikuko Fujii and Frank Lowes, which helped in making improvements on the original version. We also gratefully acknowledge the contributors to IGRF models. This study is financially supported by the National Natural Science Foundation of China (40204003 and 40074015).

\section{References}

Barton, C. E., International Geomagnetic Reference Field: The Seventh Generation, J. Geomag. Geoelectr., 49, 123-148, 1997.

Briggs, B. H., On the analysis of moving pattern in geophysics-I. Correlation analysis, J. Atmos. Terr. Phys., 30, 1777-1788, 1968a.

Briggs, B. H., On the analysis of moving pattern in geophysics-II. Dispersion analysis, J. Atmos. Terr. Phys., 30, 1789-1794, 1968 b.

Bullard, E. C., C. Freedman, H. Gellman, and J. Nixon, The westward drift of the Earth's magnetic field, Thil. Trans. Roy. Soc., A243, 67-92, 1950.

Halley, E., An account of the cause of the change of the variation of the magnetical needle; with an hypothesis of the structure of the internal parts of the Earth, Thil. Trans. Roy. Soc. Lond., 17, 563-578, 1692.

IAGA Division 5, Working Group 8, International Geomagnetic Reference Field 2000, Geophys. J. Int., 141, 259, 2000.

Jault, D., C. Gire, and J. L. Le Mouel, Westward drift, core motions and exchanges of angular momentum between core and mantle, Nature, 333, $353-356,1988$.

Langel, R. A., The Main Field, in Geomagnetism, Vol. 1, edited by J. A. Jacobs, pp. 249-512, Academic Press, London, 1987.
Malin, S. R. C., Geomagnetic secular variation and its changes, 1942.5 to 1962.5, Geophys. J. R. astr. Soc., 17, 415-441, 1969.

Malin, S. R. C. and I. Saunder, Rotation of the Earth's magnetic field, Nature, 248, 403-405, 1973

Mandea, M. and S. Macmillan, International Geomagnetic Reference Field - the eighth generation, Earth Planets Space, 52, 1119-1124, 2000 Matsushima, M. and Y. Honkura, Fluctuation of the standing and drifting parts of the Earth's magnetic field, Geophys. J., 94, 35-50, 1988.

Tank, S. B., Rotation of the geomagnetic field about an optimum pole, Geophys. J. Int., 140, 461-464, 2000.

Wei, Z.-G. and W.-Y. Xu, Westward drift of the geomagnetic anomaly in east Asia, Chinese J. Geophys., 43(1), 49-56, 2000.

Wei Z.-G. and W.-Y. Xu, Drift and intensity variations of the geomagnetic field, Chinese J. Geophys., 44(4), 496-505, 2001.

Wei Z.-G. and W.-Y. Xu, Latitude-dependence and dispersion of the westward drift in the geomagnetic field, Chinese Sci. Bull., 47(4), 330-333, 2002.

Xu, W.-Y., Unusual behaviour of The IGRF during the 1945-1955 period, Earth Planets Space, 52, 1227-1233, 2000.

$\mathrm{Xu}, \mathrm{W} .-$ Y., Revision of the high-degree Gauss coefficients in the IGRF 1945-1955 models by using natural orthogonal component analysis, Earth Planets Space, 54, 753-761, 2002.

Yukutake, T., The westward drift of the magnetic field of the earth, Bull. Earthquake Res. Inst., 40, 1-65, 1962.

Yukutake, T., The drift velocity of the geomagnetic secular variation, $J$. Geomag. Geoelectr., 20, 403-414, 1968.

Yukutake, T., A stratified core motion inferred from geomagnetic secular variations, Phys. Earth Plan. Int., 24, 253-258, 1981.

Yukutake, T. and H. Tachinaka, The westward drift of the geomagnetic secular variation, Bull. Earthquake Res. Inst., 46, 1075-1102, 1968.

Z.-G. Wei (e-mail: magstorm@mail.igcas.ac.cn) and W.-Y. Xu (e-mail: wyxu@mail.igcas.ac.cn) 